\title{
Adverse events of six COVID-19 vaccines in patients with autoimmune rheumatic diseases: a cross-sectional study
}

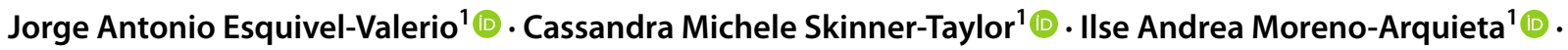 \\ Jesus Alberto Cardenas-de la Garza ${ }^{1} \mathbb{D}$. Gisela Garcia-Arellano ${ }^{1} \mathbb{D}$. Patricia Ludivina Gonzalez-Garcia ${ }^{1}$ (1) · Fatima del \\ Rocio Almaraz-Juarez ${ }^{1}$ (i) . Dioncio Angel Galarza-Delgado ${ }^{1}[$
}

Received: 11 August 2021 / Accepted: 28 September 2021 / Published online: 7 October 2021

(c) The Author(s), under exclusive licence to Springer-Verlag GmbH Germany, part of Springer Nature 2021

\begin{abstract}
Data regarding COVID-19 vaccine efficacy and adverse events (AE) in patients with autoimmune and inflammatory rheumatic diseases (AIIRD) have been published recently although these mostly include the mRNA vaccines (Pfizer-BioNTech and Moderna) and the ChAdOx1 nCoV-19/AZD1222 (Oxford-AstraZeneca). This research aimed to study the prevalence of AE presented with six different SARS-CoV-2 vaccines \{ChadOX1 nCoV-19 (AZD1222), Ad5-nCoV2, Ad26.COV2.S, mRNA-1273, BNT162b2, and CoronaVac \} in Mexican patients with AIIRD. We performed a cross-sectional study about vaccine history. Two hundred and twenty five consecutive patients were recruited, mean age was 50.7 years and the majority $(n=213 ; 94.6 \%)$ were females. One hundred and seven (47.5\%) received BNT162b2 mRNA, 34 (15.1\%) Ad5-nCoV, 29 (12.8\%) mRNA-1273, 28 (12.4\%) ChAdOX1 nCoV-19 (AZD1222), 22 (9.7\%) CoronaVac and 5 (2.2\%) Ad26.COV2.S. The vaccines that had the most AE proportionally to the number of patients vaccinated were Janssen $(5 ; 100 \%)$ followed by Pfizer-BioNTEch $(86 ; 80 \%)$ and CanSinoBIO $(27 ; 79.4 \%)$. Localized pain was the most frequent $(158 ; 70.2 \%)$ AE. Fatigue $(78 ; 34.7 \%)$, headache $(69 ; 30.6 \%)$ and muscle ache $(66 ; 29.3 \%)$ were the most common systemic symptoms. No serious AE that required medical attention or hospitalization were reported. The current results support the safety of different COVID-19 vaccines in patients with AIIRD. This information can help fight vaccine hesitancy in this population.
\end{abstract}

Keywords COVID-19 vaccines $\cdot$ SARS-CoV-2 Vaccine hesitancy $\cdot$ Autoimmune rheumatic diseases $\cdot$ Drug-related side effects and adverse reactions

\section{Introduction}

The COVID-19 pandemic continues worldwide, and some countries are already experiencing their third wave. As of September 14, 2021, 225,545,060 cases of COVID-19 and 4,644,078 deaths have been reported globally [1]. In Mexico, 3,516,043 cases of COVID-19 have been confirmed,

Jorge Antonio Esquivel-Valerio

jesquive@yahoo.com; Jorge.esquivelvl@uanl.edu.mx

Cassandra Michele Skinner-Taylor

casskinner@prodigy.net.mx

Ilse Andrea Moreno-Arquieta

ilseandrea.moreno@gmail.com

Jesus Alberto Cardenas-de la Garza

cardenasdelagarza@gmail.com

Gisela Garcia-Arellano

gga2788@hotmail.com and 267,969 deaths have been declared as of this same date [2]. The symptoms and disease severity of COVID-19 vary greatly from asymptomatic individuals to respiratory failure and death. Common symptoms include fever, cough, myalgias, diarrhea, anosmia, ageusia and dyspnea [3, 4].

COVID-19 vaccination prevents infection, reduces disease severity, and may prevent person-to-person

Patricia Ludivina Gonzalez-Garcia ludivina.gonzalezgrc@uanl.edu.mx

Fatima del Rocio Almaraz-Juarez rocio.almaraz@icloud.com

Dioncio Angel Galarza-Delgado dgalarza@medicinauanl.mx

1 Rheumatology Service, University Hospital "Dr. Jose Eleuterio Gonzalez", Universidad Autónoma de Nuevo León, Av. Gonzalitos y Av. Madero s/n, Colonia Mitras Centro, C.P. 64460 Monterrey, NL, Mexico 
transmission [5]. Despite this, vaccine hesitancy remains an alarming problem worldwide. Data regarding COVID-19 vaccine efficacy and adverse events (AE) in patients with autoimmune and inflammatory rheumatic diseases (AIIRD) have been published recently although these mostly include the mRNA vaccines (Pfizer-BioNTech and Moderna) and the ChAdOx1 nCoV-19/AZD1222 (Oxford/AstraZeneca) [6-9]. Many other vaccines are being administered worldwide and data in AIIRD population are limited.

In Mexico, many different vaccines are approved and administered including BNT162b2 (Pfizer-BioNTech), ChAdOx1 nCoV-19/AZD1222 (Oxford-AstraZeneca), Ad26.COV2.S (Janssen), Ad5-nCoV (CanSinoBIO), Coronavac (Sinovac), BBV152 COVAXIN (Bharat Biotech India), mRNA-1273 (Moderna), and Sputnik V (The Gamaleya National Center). This research aimed to study the prevalence of AE presented with six different SARS-CoV-2 vaccines (ChadOX1 nCoV-19/AZD1222, Ad5-nCoV2, Ad26.COV2.S, mRNA-1273, BNT162b2 and CoronaVac) in Mexican patients with AIIRD.

\section{Methods}

We performed a cross-sectional study from May 3 to July 21, 2021 about vaccine history in patients of the outpatient rheumatology clinic of the University Hospital "Dr. José Eleuterio González", UANL. All the patients with a history of COVID-19 vaccination attending the clinic were invited to participate. All the participants were informed of the purpose of the survey and verbal consent was obtained before their inclusion. The institutional ethics and research committee approved the protocol as part of the Rheumatology Integral Care Program (No: RE 20-00013). The survey included demographic data (age, sex, rheumatic disease diagnosis), SARS-CoV-2 infection history (severity of illness, inpatient/ outpatient management), and vaccine AE (local or systemic reactions). No identifiable data were recorded. The survey contained checkboxes and open-ended questions. The survey was applied to a pilot group of ten patients to ensure clarity. Patients without AIIRD, without COVID-19 vaccination, or who could not recall the vaccine administered were excluded. Statistical analyses were done with IBM SPSS v.23 (IBM Inc., Armonk, NY, USA).

\section{Results}

We recruited 225 consecutive patients. Mean age was 50.7 years and the majority $(n=213 ; 94.6 \%)$ were females. One hundred thirty two $(58.6 \%)$ had rheumatoid arthritis, $25(11.1 \%)$, systemic lupus erythematosus, 22 (9.7\%) axial spondyloarthritis, 12 (5.3\%) primary Sjögren's syndrome,
8 (3.5\%) inflammatory myopathies, and $25(11.1 \%)$ others. Forty-two (18.5\%) patients had COVID-19 of which 29 (69\%) had mild symptoms while $12(28.6 \%)$ moderate and only $1(2.4 \%)$ severe. A total of 5 (11.9\%) subjects required inpatient treatment.

One hundred and seven $(47.5 \%)$ received BNT162b2 mRNA, 34 (15.1\%) Ad5-nCoV, 29 (12.8\%) mRNA-1273, 28 (12.4\%) ChAdOX1 nCoV-19 (AZD1222), 22 (9.7\%) CoronaVac and 5 (2.2\%) Ad26.COV2.S. None of the patients referred vaccination with Sputnik V. The vaccines that had the most AE proportionally to the number of patients vaccinated were Janssen $(5 ; 100 \%)$ followed by Pfizer-BioNTech (86; 80\%) and CanSinoBIO (27; 79.4\%). Localized pain was the most frequent $(158 ; 70.2 \%)$ AE. Fatigue $(78 ; 34.7 \%)$, headache $(69 ; 30.6 \%)$ and muscle ache $(66 ; 29.3 \%)$ were the most common systemic symptoms. Fifty-two (23.1\%) patients did not present any AE. None of the symptoms was severe enough to require medical attention or hospitalization (Table 1).

\section{Discussion}

Vaccine hesitancy is defined as the "delay in acceptance or refusal of vaccination despite availability of vaccination services" [10]. Vaccine hesitancy is a complex phenomenon that actively threatens global health. Regarding the COVID19 vaccines, the rates of vaccines acceptance and vaccine hesitancy greatly vary among populations and countries. A review of low- and middle-income countries found a COVID-19 vaccine acceptance rate of $80.3 \%$ compared to $64 \%$ in the United States and 30.4\% in Russia (classified as an upper-middle-income country) [11]. In Latin America, COVID-19 vaccine intention rates are high in Mexico (88.4\%) and Brazil (83.1\%), two countries with a high case and mortality rate, and low in Haiti (43.2\%) and Paraguay (64.6\%) [12]. Despite the high vaccination intention rates, fear to $\mathrm{AE}$ of the COVID-10 vaccine rates is very high in Latin America (81.2\%) [12].

Recent reports have analyzed the vaccine intention and hesitancy in patients with autoimmune rheumatic diseases. In Italy, the acceptance rate for COVID-19 vaccination in a sample of 344 patients with rheumatic and musculoskeletal diseases was 54.9\% [13]. Studies from India and Turkey reported an acceptance rate of $54 \%$ and $29.2 \%$, respectively $[14,15]$. One of the most frequent reasons for vaccine hesitancy in patients with autoimmune rheumatic diseases is fear or concern to vaccine $\mathrm{AE}[14,15]$. Reports of $\mathrm{AE}$ from different vaccines in patients with AIIRD may help tackle this alarming problem worldwide.

Information regarding AE of COVID-19 vaccination in patients with AIIRD has been increasing. The majority of case series analyze the vaccines most commonly employed 
Table 1 Results of adverse events (AE) in each vaccine against SARS-COV-2

\begin{tabular}{|c|c|c|c|c|c|c|}
\hline \multirow[t]{2}{*}{ Adverse events } & $\begin{array}{l}\text { ChadOX1 nCoV-19 } \\
\text { (AZD1222) } \\
\text { (Oxford-AstraZen- } \\
\text { eca) }\end{array}$ & $\begin{array}{l}\text { Ad5-nCoV2 } \\
\text { (CanSinoBIO) }\end{array}$ & $\begin{array}{l}\text { Ad26.COV2.S } \\
\text { (Janssen) }\end{array}$ & $\begin{array}{l}\text { mRNA-1273 } \\
\text { (Moderna) }\end{array}$ & $\begin{array}{l}\text { BNT162b2 } \\
\text { (Pfizer-BioN- } \\
\text { Tech) }\end{array}$ & $\begin{array}{l}\text { CoronaVac } \\
\text { (Sinovac) }\end{array}$ \\
\hline & $28(12)$ & $34(14.6)$ & $5(2.1)$ & $29(12.5)$ & $107(46.1)$ & $22(9.5)$ \\
\hline Local $n(\%)$ & $17(60.7)$ & $27(79.4)$ & $5(100)$ & $20(69)$ & $81(75.7)$ & $8(36.4)$ \\
\hline Pain & $17(100)$ & $27(79.4)$ & $5(100)$ & $20(69)$ & $81(75.7)$ & $8(36.3)$ \\
\hline Redness & $2(7.1)$ & $1(2.9)$ & $1(20)$ & $3(10.3)$ & $9(8.4)$ & $0(0)$ \\
\hline Swelling & $1(3.5)$ & $1(2.9)$ & $1(20)$ & $4(13.7)$ & $10(9.3)$ & $0(0)$ \\
\hline Systemic $n(\%)$ & $14(50)$ & $20(58.8)$ & $4(80)$ & $13(44.8)$ & $49(45.8)$ & $7(31.8)$ \\
\hline Headache & $10(35.7)$ & $11(32.3)$ & $3(60)$ & $8(27.5)$ & 34 (31.7) & $3(13.6)$ \\
\hline Muscle ache & $9(32.1)$ & $11(32.3)$ & $3(60)$ & $7(24.1)$ & $32(29.9)$ & $4(18.1)$ \\
\hline Fever & $4(14.2)$ & $7(20.5)$ & $1(20)$ & $3(10.3)$ & $9(8.4)$ & $1(4.5)$ \\
\hline Chills & $3(10.7)$ & $2(5.8)$ & $3(60)$ & $4(13.7)$ & $18(16.8)$ & $2(9)$ \\
\hline Fatigue & $14(50)$ & $12(35.2)$ & $3(60)$ & $6(20.6)$ & $40(37.3)$ & $3(13.6)$ \\
\hline Nausea & $3(10.7)$ & $2(5.8)$ & $1(20)$ & $1(3.4)$ & $9(8.4)$ & $2(9)$ \\
\hline Abdominal pain & $0(0)$ & $2(5.8)$ & $1(20)$ & $3(10.3)$ & $8(7.4)$ & $0(0)$ \\
\hline Leg pain & $5(17.8)$ & $5(14.7)$ & $2(40)$ & $1(3.4)$ & $11(10.2)$ & $2(9)$ \\
\hline Dyspnea & $1(3.5)$ & $0(0)$ & $0(0)$ & $1(3.4)$ & $1(0.9)$ & $1(4.5)$ \\
\hline $\begin{array}{l}\text { Total of patients with } \\
\text { AE } n(\%)\end{array}$ & $22(78.5)$ & $27(79.4)$ & $5(100)$ & $21(72.4)$ & $86(80)$ & $12(54.5)$ \\
\hline
\end{tabular}

in Western Europe and the United States particularly PfizerBioNTech, Oxford-AstraZeneca and Moderna vaccines. The adverse event profile of these vaccines has been very similar to the general population and severe adverse events have been very rare $[7,9,16,17]$. Adverse event data from other vaccines in rheumatic patients predominantly employed in Asia, Latin America, and Eastern Europe are very limited. In our case series of 225 patients presented herein, none experienced severe $\mathrm{AE}$ that required medical attention or hospitalization. The findings are reassuring and accordant with other case series $[9,16]$.

Our study has several limitations including the crosssectional design, sample size, descriptive statistical analysis, and lack of survey validation or reliability testing [18]. Additionally, long-term adverse events were not evaluated, and all the subjects included had received the vaccines in the previous 6 months. Strengths include the number of vaccines included and the participation of Latin American Hispanic patients, a population often underrepresented in the literature.

\section{Conclusion}

The current results support the safety of different COVID-19 vaccines in patients with AIIRD. No serious $\mathrm{AE}$ that required medical attention or hospitalization were reported. This information can help fight vaccine hesitancy in this population.
More studies with a higher number of patients and long-term follow-up are needed to improve the current knowledge on the safety of these vaccines in AIIRD.

Author contributions All authors contributed to the study conception and design. Recruitment was performed by IAMA, PLGO, and FdRA-J. Analysis and interpretations were performed by JAEV, IAMA, PLGO, and FdRA-J. Writing of the first draft was performed by JACdlG, IAMA, and GGA. All the authors read and approved the final manuscript.

Funding No external funding was received.

Data availability The authors confirm that the data supporting the findings of this study are available within the article.

\section{Declarations}

Conflict of interest None declared.

Ethical approval The institutional ethics and research committee approved the protocol as part of the Rheumatology Integral Care Program (No: RE 20-00013).

Consent to participate Verbal consent was obtained from the patients.

Consent to publication Patient consent for publication not required. 


\section{References}

1. Johns Hopkins University (2021) COVID-19 dashboard. https:// coronavirus.jhu.edu. Accessed 14 Sep 2021.

2. Johns Hopkins University (2021) Coronavirus resource center Mexico. https://coronavirus.jhu.edu/region/mexico. Accessed 14 Sep 2021.

3. da Rosa MR, Francelino Silva Junior LC, Santos Santana FM et al (2021) Clinical manifestations of COVID-19 in the general population: systematic review. Wien Klin Wochenschr 133:377-382. https://doi.org/10.1007/s00508-020-01760-4

4. Gandhi RT, Lynch JB, Del Rio C (2020) Mild or moderate Covid19. N Engl J Med 383:1757-1766. https://doi.org/10.1056/NEJMc p2009249

5. Mostaghimi D, Valdez CN, Larson HT, Kalinich CC, Iwasaki A (2021) Prevention of host-to-host transmission by SARS-CoV-2 vaccines. Lancet Infect Dis. https://doi.org/10.1016/S14733099(21)00472-2

6. Braun-Moscovici Y, Kaplan M, Braun M et al (2021) Disease activity and humoral response in patients with inflammatory rheumatic diseases after two doses of the Pfizer mRNA vaccine against SARS-CoV-2. Ann Rheum Dis 80:1317-1321. https://doi.org/10. 1136/annrheumdis-2021-220503

7. Cherian S, Paul A, Ahmed S et al (2021) Safety of the ChAdOx1 nCoV-19 and the BBV152 vaccines in 724 patients with rheumatic diseases: a post-vaccination cross-sectional survey. Rheumatol Int 41(8):1441-1445. https://doi.org/10.1007/s00296-021-04917-0

8. Connolly CM, Ruddy JA, Boyarsky BJ et al (2021) Safety of the first dose of mRNA SARS-CoV-2 vaccines in patients with rheumatic and musculoskeletal diseases. Ann Rheum Dis. https://doi. org/10.1136/annrheumdis-2021-220231

9. Furer V, Eviatar T, Zisman D et al (2021) Immunogenicity and safety of the BNT162b2 mRNA COVID-19 vaccine in adult patients with autoimmune inflammatory rheumatic diseases and in the general population: a multicentre study. Ann Rheum Dis 80:1330-1338. https://doi.org/10.1136/annrheumdis-2021-220647

10. MacDonald NE, SAGE Working Group on Vaccine Hesitancy (2015) Vaccine hesitancy: definition, scope and determinants. Vaccine 33:4161-4164
11. Solis Arce JS, Warren SS, Meriggi NF et al (2021) COVID-19 vaccine acceptance and hesitancy in low- and middle-income countries. Nat Med 27:1385-1394. https://doi.org/10.1038/ s41591-021-01454-y

12. Urrunaga-Pastor D, Bendezu-Quispe G, Herrera-Anazco P et al (2021) Cross-sectional analysis of COVID-19 vaccine intention, perceptions and hesitancy across Latin America and the Caribbean. Travel Med Infect Dis 41:102059. https://doi.org/10.1016/j. tmaid.2021.102059

13. Priori R, Pellegrino G, Colafrancesco S et al (2021) SARS-CoV-2 vaccine hesitancy among patients with rheumatic and musculoskeletal diseases: a message for rheumatologists. Ann Rheum Dis 80:953-954. https://doi.org/10.1136/annrheumdis-2021-220059

14. Gaur P, Agrawat H, Shukla A (2021) COVID-19 vaccine hesitancy in patients with systemic autoimmune rheumatic disease: an interview-based survey. Rheumatol Int 41:1601-1605. https:// doi.org/10.1007/s00296-021-04938-9

15. Yurttas B, Poyraz BC, Sut N et al (2021) Willingness to get the COVID-19 vaccine among patients with rheumatic diseases, healthcare workers and general population in Turkey: a webbased survey. Rheumatol Int 41:1105-1114. https://doi.org/10. 1007/s00296-021-04841-3

16. Bartels LE, Ammitzboll C, Andersen JB et al (2021) Local and systemic reactogenicity of COVID-19 vaccine BNT162b2 in patients with systemic lupus erythematosus and rheumatoid arthritis. Rheumatol Int. https://doi.org/10.1007/s00296-021-04972-7

17. Sattui SE, Liew JW, Kennedy K et al (2021) Early experience of COVID-19 vaccination in adults with systemic rheumatic diseases: results from the COVID-19 global rheumatology alliance vaccine survey. RMD Open 7:e001814. https://doi.org/10.1136/ rmdopen-2021-001814

18. Gaur PS, Zimba O, Agarwal V, Gupta L (2020) Reporting survey based studies—a primer for authors. J Korean Med Sci 35:398. https://doi.org/10.3346/jkms.2020.35.e398

Publisher's Note Springer Nature remains neutral with regard to jurisdictional claims in published maps and institutional affiliations. 